\title{
Roles for the Dorsal Striatum in Aversive Behavior
}

\author{
Adrien T. Stanley ${ }^{1}$, Pellegrino Lippiello ${ }^{2}$, David Sulzer ${ }^{3,4 *}$ and Maria Concetta Miniaci ${ }^{2 *}$ \\ ${ }^{1}$ Departments of Biology and Psychiatry, Columbia University, New York, NY, United States, ${ }^{2}$ Department of Pharmacy, \\ School of Medicine, University of Naples Federico II, Naples, Italy, ${ }^{3}$ Departments of Psychiatry, Neurology, and \\ Pharmacology, Columbia University Medical Center, New York, NY, United States, ${ }^{4}$ Division of Molecular Therapeutics, \\ New York State Psychiatric Institute, New York, NY, United States
}

The ability to identify and avoid environmental stimuli that signal danger is essential to survival. Our understanding of how the brain encodes aversive behaviors has been primarily focused on roles for the amygdala, hippocampus (HIPP), prefrontal cortex, ventral midbrain, and ventral striatum. Relatively little attention has been paid to contributions from the dorsal striatum (DS) to aversive learning, despite its well-established role in stimulus-response learning. Here, we review studies exploring

OPEN ACCESS

Edited by:

Maurizio Giustetto,

University of Turin, Italy

Reviewed by:

Alfonso Junior Apicella,

University of Texas at San Antonio,

United States

Sevil Duvarci,

Goethe University Frankfurt, Germany

*Correspondence: Maria Concetta Miniaci maria.miniaci@unina.it David Sulzer

ds43@cumc.columbia.edu

Specialty section:

This article was submitted to

Cellular Neurophysiology,

a section of the journal

Frontiers in Cellular Neuroscience

Received: 27 November 2020 Accepted: 25 January 2021 Published: 16 February 2021

Citation:

Stanley AT, Lippiello P, Sulzer D and Miniaci MC (2021) Roles for the Dorsal Striatum in Aversive Behavior. Front. Cell. Neurosci. 15:634493. doi: 10.3389/fncel.2021.634493 the role of DS in aversive learning, including different roles for the dorsomedial and dorsolateral striatum in Pavlovian fear conditioning as well as innate and inhibitory avoidance (IA) behaviors. We outline how future investigation might determine specific contributions from DS subregions, cell types, and connections that contribute to aversive behavior.

Keywords: dorsal striatum, fear conditioning, inhibitory avoidance, aversion, threat

\section{INTRODUCTION}

The ability to learn associations between environmental stimuli and aversive events is essential to survival, as it allows the organism to avoid these events and reduce the chance of harm. In a threatening context, individuals may use defense strategies, such as the fight or flight response, or passive coping, characterized by immobility and withdrawal (Koolhaas et al., 1999; Wood and Bhatnagar, 2015). However, aberrant avoidance responses can lead to psychiatric diseases, including anxiety and post-traumatic stress disorders (PTSD; Sripada et al., 2013).

Pavlovian fear conditioning paradigms in rodents have served as the most widely used approaches for the analysis of neural mechanisms of learning and memory-related to aversive stimuli (LeDoux, 2000; Maren, 2001; Cefaliello et al., 2020). During fear conditioning, animals learn to associate a neutral conditioned stimulus (CS) that can either be a temporally discrete sensory event or cues, such as a light or tone, or the environmental context itself with an aversive event such as an inescapable electric foot-shock unconditioned stimulus (US), so that the cue becomes a threatening signal and elicits conditioned reactions such as freezing and tachycardia. Both context and cue-dependent fear conditioning paradigms are learned rapidly and elicit robust memory. An aversive stimulus can also elicit actions that avoid the upcoming threat, e.g., in active avoidance conditioning, the animal learns to avoid a shock.

Much has been learned about the neural basis of fear learning and memory through studies of fear conditioning. Studies in both humans and rodents have corroborated 
a central role for the amygdala in the acquisition and storage of conditioned fear (Nader et al., 2001; Phelps and LeDoux, 2005). In particular, sensory inputs from the cortex and thalamus enter the amygdala via the basolateral amygdala (BLA). This sensory input, as well as reciprocal input from the ventral hippocampus (HIPP) and medial prefrontal cortex (MPFC), plays an important role in encoding CS-US association. In response to CS, the BLA will activate central amygdala (CEA) nuclei which in turn sends projections to the bed nucleus of the stria terminalis, periaqueductal gray (PAG), and the hypothalamus driving the expression of behavioral and autonomic responses (LeDoux et al., 1988; Tovote et al., 2015; Krabbe et al., 2018; Sah et al., 2020; Figure 1A).

Active avoidance learning proceeds through three distinct phases, involving different neural circuits (Cain and LeDoux, 2007; LeDoux et al., 2017). The first phase consists of CSUS fear conditioning (i.e., the animals learn that a stimulus predicts a threat) and involves the BLA-CeA-PAG pathway mentioned above. The second phase involves action-outcome learning (i.e., animals learn to perform actions that terminate the CS) and depends on the activation of the basolateral amygdalanucleus accumbens pathway; the infralimbic prefrontal cortex is also recruited to suppresses the CeA-mediated freezing and facilitate avoidance (Moscarello and LeDoux, 2013). According to LeDoux, avoidance may be reinforced not only by the removal of danger (negative reinforcement) but also by the addition of a safety cue (positive reinforcement; LeDoux et al., 2017). In the third phase, avoidance behavior becomes habitual (i.e., outcome independent) and likely involves the dorsal striatum (DS).

The DS has been extensively studied for its essential role in stimulus-response learning, i.e., a process by which a sensory cue can elicit a consistent motor response (Packard and Knowlton, 2002). Whether the DS contributes to the aversive learning process is not fully understood.

Various aversive stimuli such as noxious heat, cold, mechanical, and electrical stimuli alter the activity of the DS, and many studies report that alterations of DS function using lesions and microinjections affect pain perception (Chudler and Dong, 1995; Borsook et al., 2010). Interestingly, patients with Parkinson's disease, a movement disorder mostly due to decreased dopamine release in the DS, often present with comorbid chronic pain (Buhidma et al., 2020). Chronic pain is also co-morbid with PTSD (Fishbain et al., 2017), and processing of aversive stimuli within the DS is important for pain-avoidance learning (Koyama et al., 2000).

This review highlights studies that investigate the role of the DS in aversive learning with a focus on fear conditioning and avoidance.

\section{NEURONAL PROJECTIONS FROM AND TO THE DORSAL STRIATUM}

The DS is composed of a medial portion (DMS), a lateral portion (DLS), and a posterior portion (tail of the striatum, TS), each with distinct functions and inputs (Hunnicutt et al., 2016). The DMS primarily receives inputs from limbic and association cortices such as anterior cingulate cortex, parietal association cortex, prelimbic cortex (LC), and infralimbic cortex while the DLS receives inputs primarily from the motor and somatosensory cortices such as $\mathrm{M} 1 / 2$ and $\mathrm{S} 1 / 2$. The TS primarily receives information from sensory cortices, especially the auditory and visual cortex (Haber, 2003; Hunnicutt et al., 2016). All of these regions receive dopaminergic input from the substantia nigra compacta $(\mathrm{SNc})$, which plays an important role in synaptic plasticity (Arbuthnott et al., 2000). Dopaminergic projections from the lateral $\mathrm{SNc}$ project exclusively to the TS while all other portions of the $\mathrm{SNc}$ project throughout the DS (Beckstead et al., 1979).

Several regions that project to the DS subdivisions are implicated in fear learning, including glutamatergic excitatory direct projections from the BLA and $\mathrm{MPFC}$, and indirectly, GABergic inhibitory inputs from the CeA (Conzales and Chesselet, 1990; Hunnicutt et al., 2016; Figure 1A). As does the amygdala, the DS receives input from the thalamus, sensory cortices, and association cortices (LeDoux, 2000; Hunnicutt et al., 2016; Figure 1B). The cortical and thalamic

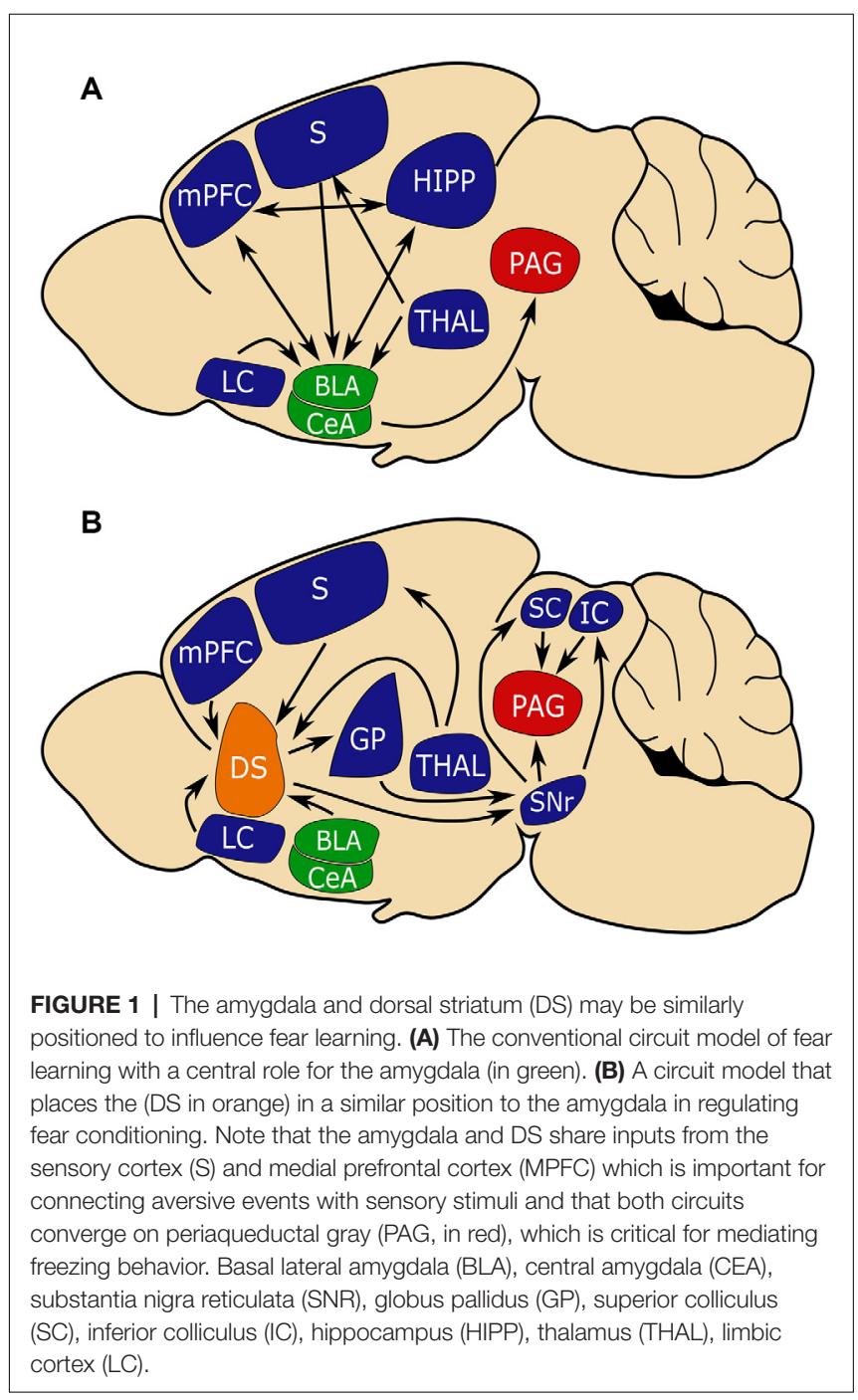


inputs to the amygdala are critical for associating sensory stimuli with aversive events, and it may be that similar inputs to the DS serve complementary functions (Ponvert and Jaramillo, 2019).

The DS is predominantly composed of spiny projection neurons (SPNs) that mostly express either D1 dopamine receptors or D2 receptors. D1 SPNs project directly to the substantia nigra reticulata $(\mathrm{SNr})$ and are referred to as direct pathway SPNs. D1 SPN projections to the $\mathrm{SNr}$ are topographically organized with D1 SPNs of the anterior DS project medially, while D1 SPNs of the posterior DMS/DLS and TS project laterally (Hedreen and Delong, 1991). D2 SPNs indirectly project to the $\mathrm{SNr}$ via the globus pallidus (GP) externa (GPe) and subthalamic nuclei and are thus referred to as the indirect pathway (DeLong, 1990; Bertran-Gonzalez et al., 2010). D2 SPN projection to the GPe is also topographically organized with D2 SPNs of anterior DS projecting anteriorly, while D2 SPNs of the posterior DS project posteriorly (Hedreen and Delong, 1991).

Activation of the two SPN populations often elicits opposite effects on behavior (Lenz and Lobo, 2013). Both the GPe and $\mathrm{SNr}$ have been shown to play roles in aversive behaviors (Ipser et al., 2013; Hormigo et al., 2016; Almada et al., 2018). The SNr projects to several regions involved in the fear response including the superior colliculus (SC), inferior colliculus (IC), and PAG (Castellan-Baldan et al., 2006). Together, these pathways place the DS in a position to integrate sensory and aversive information as well as modulate aversive responses.

\section{DORSAL STRIATUM AND FEAR CONDITIONING}

Both cued fear and contextual fear conditionings are composed of three distinct phases: acquisition, consolidation, and memory recall.

Brain regions involved in fear conditioning are differentially engaged by cued vs. contextual responses, as well as during different stages of the conditioning process. For example, the hippocampus has been shown to play a pivotal role in the acquisition and consolidation of contextual fear learning with little involvement in cued fear (Sanders et al., 2003), while the amygdala plays roles in both forms and during all phases of fear conditioning (LeDoux, 2000; Tovote et al., 2015). A summary of activity changes and the consequences of manipulating the DS function are outlined in Figures 2A,B.

\section{Cued Fear Conditioning}

Cued fear conditioning paradigms involve placing an animal in a chamber with metal bar floors, and after a habituation period, presenting the mouse an auditory CS and an electrical foot-shock (US) that temporally overlaps with the CS. To test the strength of the CS-US association, during the recall phase, the mouse is placed in a new context and freezing responses to the CS are measured.

Functional magnetic resonance imaging and positron emission tomography (PET) studies in humans demonstrate that the putamen (corresponding to the mouse DLS), but not caudate (corresponding to rodent DMS), is activated during
A

Cue fear conditioning

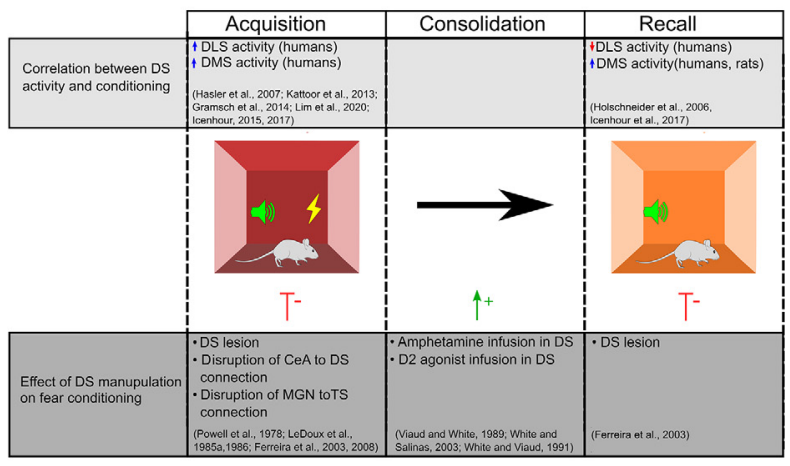

B

Context fear conditioning

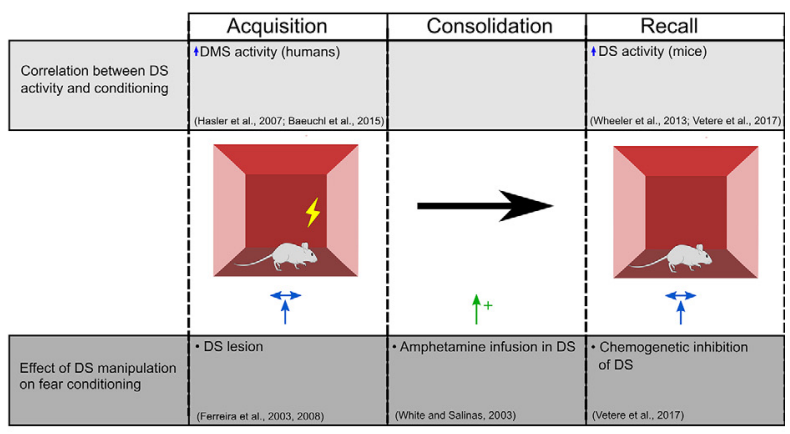

C

Avoidance behaviour

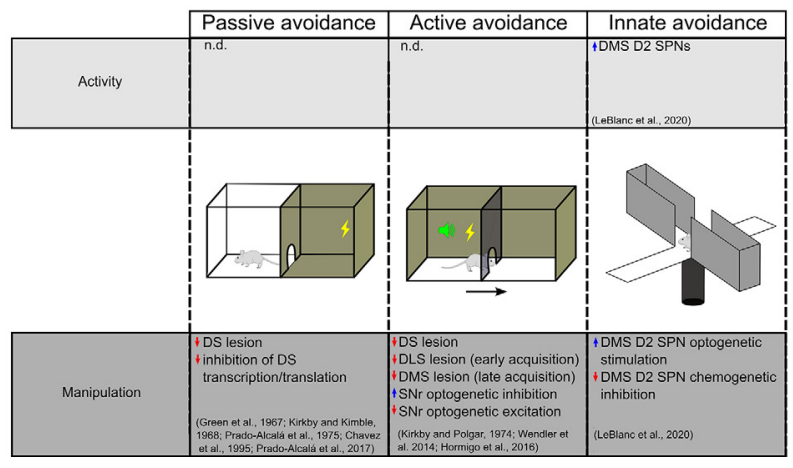

FIGURE 2 | Evidence for DS's role in aversive behavior. (A) The upper chart highlights studies that demonstrate a correlation between DS activity during different stages of cue fear conditioning. The lower chart highlights studies on the effect of DS manipulations on cue fear learning. (B) Studies examining the effect of context fear conditioning on DS activity (top) and the effect of DS manipulations on context fear learning (bottom). Central amygdala (CEA), medial geniculate nucleus (MGN), dorsal striatum (DS), dorsal medial striatum (DMS), dorsolateral striatum (DLS), the tail of the striatum (TS). (C) Evidence for DS's role in inhibitory and innate avoidance. Dorsal striatum (DS), dorsal medial striatum (DMS), dorsolateral striatum (DLS), substantia nigra reticulata (SNR)

the acquisition phase of fear conditioning (Hasler et al., 2007; Kattoor et al., 2013; Gramsch et al., 2014). However, further studies in humans show that the caudate is also active during both acquisition and recall (Icenhour et al., 2015, 2017). An 
increase in DMS activity has also been demonstrated in rats during cued fear recall, in association with a decrease in the DLS blood flow (Holschneider et al., 2006). Based on these studies, we may argue that there is a differential contribution of DLS and DMS in fear acquisition and recall but conflicts remain to be addressed.

To determine the causal relationship between striatal activity and cued fear learning, selective lesions and pharmacological inactivation studies have been performed. For example, in rats, blocking DS function by an electrolytic lesion or inhibition with the $\mathrm{GABA}_{\mathrm{A}}$ agonist, muscimol, before training or before the recall, reduced freezing responses to the cue (Ferreira et al., 2003, 2008). Similarly, lesion of the DS in rabbits impairs corneo-retinal responses to learned fear cues (Powell et al., 1978).

At odds with the results of studies indicating a role for DS conditioning in fear learning is a study that compared the effect of selective lesions to the DLS, DMS, or ventral striatum on cued fear conditioning in rats, that concluded that only lesions to the ventral striatum decreased freezing during training and recall (Wendler et al., 2014). We note however that rather than measuring freezing during CS presentation, the investigators measured freezing during the entire recall session which took place in the same context as the training session, and so did not separate freezing response generated by context from that of the cue.

More broadly, a major caveat of brain lesioning studies is that they do not disentangle the contributions of the DS to acquisition vs. consolidation or recall, as these manipulations affect all phases of fear conditioning. A related issue with classical lesion studies is that they indiscriminately destroy all of the cells in a region of the DS, and so cannot identify neuronal populations that serve different or even opposing functions during fear conditioning.

Pharmacological manipulations also implicate the DS in cued fear conditioning. Selective infusion of amphetamine, an agent that drives the release of dopamine and other monoamines, during the consolidation phase enhanced fear responses during recall (Viaud and White, 1989; White and Salinas, 2003). This effect was replicated with an infusion of a D2 agonist, but not a D1 agonist, into the DS (White and Viaud, 1991). These results suggest a differential involvement of D1 and D2 receptors in cued fear consolidation. Since D2 receptors are expressed on SPNs and GABAergic and cholinergic interneurons as well as dopaminergic afferents (Fisher et al., 1994), it will be important to examine the contributions of these cell types on fear consolidation.

Disrupting the connections between DS and the other brain areas involved in fear learning can also influence fear response. Studies in rats have examined the consequences of severing the connections between the CeA and DS in fear learning (Ferreira et al., 2008). Because the CeA connects to the DS via an indirect and exclusively ipsilateral projection to the SNc (Conzales and Chesselet, 1990), the authors performed asymmetrical lesions, consisting of a unilateral CeA lesion combined with a contralateral DS lesion, while leaving the contralateral sides functional. They found that this manipulation before training, but not before recall, impaired the freezing response.

The same ablation approach in rats revealed that projections from the medial geniculate nucleus (MGN) of the thalamus to TS are important for cued fear learning (LeDoux et al., 1985a, 1986). The MGN is a portion of the thalamus that relays auditory information from the IC to the TS as well as the amygdala and auditory cortex (LeDoux et al., 1984, 1985b) Bilateral lesion of MGN before training impairs freezing during recall. This effect is replicated by unilateral ablation of MGN combined with the contralateral lesion of TS or amygdala (LeDoux et al., 1985a) These results suggest that auditory information from MGN to the TS/amygdala is essential for cued fear learning.

What role is the DS playing in cued fear learning? These studies have shown that DS activity and cued fear learning are correlated and causally related. However, because studies exploring the causal relationship rely on lesions and pharmacological manipulations that affect many subregions and cell types within the DS, it has been difficult to determine the role of the DS. Further investigations using techniques with cell-specific and region-specific targeting are needed to strengthen the evidence of the causal relation of DS activity and cued fear learning.

\section{Contextual Fear Conditioning}

Contextual fear conditioning paradigms consist of placing an animal in a chamber with metal bar floors where it is administered an electric foot-shock. In this paradigm, the foot-shock is a US and the environmental context of the conditioning chamber is the CS. To test the degree of CS-US association, during the recall phase, mice are returned to the conditioning chamber and the time spent freezing is recorded.

PET imaging in humans using $[\mathrm{O}-15] \mathrm{H}_{2} \mathrm{O}$ has revealed that the caudate is activated during the acquisition of contextual fear conditioning (Hasler et al., 2007; Baeuchl et al., 2015). A role for the DS is further supported by experiments in mice, where recall of contextual fear has been shown to increase DS expression of the immediate-early gene cFos, an indicator of neural activity (Wheeler et al., 2013; Vetere et al., 2017). In contrast, rats that naturally have a low response to contextual fear conditioning have higher mRNA expression of striatal NMDA subunit NR2A (Schenberg et al., 2006); given a requirement of NR2A for long term potentiation in the DS (Li et al., 2009), this finding suggests that enhanced plasticity within the DS may attenuate contextual fear learning. Studies investigating the causal relationship of DS activity and plasticity on contextual fear learning may shed light on this issue.

Studies selectively ablating the DS before training show no effect on contextual fear learning (Ferreira et al., 2008). Similarly, a study using chemogenetic inhibition of DS after contextual fear training showed no effect on freezing during recall (Vetere et al., 2017). On the other hand, infusion of amphetamine selectively into the DS of rats during the consolidation phase enhances the freezing during recall of contextual fear (White and Salinas, 2003), suggesting that dopamine in the DS promotes 
consolidation of contextual fear. How the dopamine, and eventually the D1 and D2 SPNs are involved in contextual fear learning remains an open question.

While these studies indicate that the DS may play a selective role in the consolidation of contextual fear, controversies remain and there are many gaps in our knowledge. Additional studies are, therefore, required that target the DS during acquisition and recall.

\section{DORSAL STRIATUM AND AVOIDANCE BEHAVIOR}

In the inhibitory avoidance (IA) test, animals learn to avoid an environment where they have previously been exposed to an aversive stimulus (US, such as a foot-shock) by remaining in the brightly lit side of a two-compartment chamber (passive avoidance paradigm) or by running into the opposite side compartment following a CS presentation (active avoidance paradigm). In this paradigm, the animals have to suppress their innate avoidance behavior i.e., their tendency to avoid bright and open areas.

A role for the DS in IA has been known since the 1960s. Several studies in rodents demonstrated that electrolytic or chemical disruption of the caudate nucleus impairs the acquisition and the retention of the passive IA task (Green et al., 1967; Kirkby and Kimble, 1968; Prado-Alcalá et al., 1975; Chavez et al., 1995; Figure 2C). A marked deficit of consolidation of a passive IA task has also been observed following inhibition of transcription and translation in DS (Prado-Alcalá et al., 2017). Interestingly, while foot-shock alone does not change the numbers of dendritic spines of SPNs in the DLS and DMS, intense IA training induces a significant increase in spine density that is proportional to the foot-shock intensity. The learninginduced increase of dendritic spines in DS may represent a cellular mechanism underlying the consolidation and persistence of memory storage (Bello-Medina et al., 2016).

Lesion studies have also demonstrated that DS has an important role in the active avoidance task (Kirkby and Polgar, 1974; Figure 2C). Wendler et al. (2014) have demonstrated a differential involvement of DS subregions since lesions of DLS impaired early acquisition whereas DMS lesion impaired the late phases of learning and the extinction. The precise striatal circuit that results in active avoidance behavior remains elusive. Using chemogenetics and optogenetics, Hormigo and collaborators (Hormigo et al., 2016) recently found that the SNr, the main DS output, modulates the avoidance response to the CS. Indeed, inhibition of SNr firing facilitates active avoidance during tone presentation while SNr excitation blocks the response. According to their putative circuit model, the CS-driven avoidance behavior may engage a multisynaptic loop that originates within and returns to the superior colliculus via the $\mathrm{SNr}$ (Hormigo et al., 2016). The SC detects sensory stimuli and sends information to the striatum via the posterior intralaminar nucleus (Linke, 1999; Krout et al., 2001). Once activated by CS, the striatal direct pathway SPNs may suppress the SNr and consequently disinhibit its projections to the SC and the mesencephalic locomotor central pattern generators (MacKay-Lyons, 2002), thus promoting active avoidance behavior.

Elevated plus maze and open field are commonly used to assess the innate tendency of mice to avoid brightly lit and open spaces such as the open arms of a maze or the center area of an open field. Evidence is now emerging that the DMS, and in particular the D2R-expressing SPNs, plays an important role in innate avoidance behavior in the elevated plus-maze and open-field behavioral tests (Figure 2C). Using fiber photometry, LeBlanc et al. (2020) demonstrated that D2 SPNs activity increases as mice enter anxiogenic areas of these tasks. Also, they demonstrated that optogenetic stimulation of D2 SPNs promotes avoidance of open areas in both tasks, while the chemogenetic inhibition of D2 SPNs reduces avoidance behavior. Interestingly, knocking out the D2R on cholinergic interneurons or dopamine neurons did not affect the mice's performance in the exploratory tasks, suggesting that the DMS indirect pathway neurons are critical for evoking aversive behavior.

\section{CONCLUSIONS AND DIRECTIONS FOR FUTURE RESEARCH}

While it is well established that the DS facilitates reward-based stimulus-response learning (Packard and Knowlton, 2002), there is far less evidence that this region mediates the freezing or escape responses to aversive stimuli. Our review of current data indicates that the patterns for appetitive learning are different from those associated with aversive instrumental conditioning and strongly supports a role for the DS in cued fear conditioning and avoidance learning, while there are conflicting findings of its involvement in contextual fear.

A fundamental difference between the learning paradigms seems to reflect the participation of different DS regions. In appetitive instrumental conditioning, the goal-directed action is quickly acquired and involves DMS activation, while the habit component is learned slowly and is apparently mediated by the DLS (Yin et al., 2006). This suggests that the DMS is required for the early stages of appetitive learning, while the DLS is involved in later stages.

Aversive learning appears to engage DS subregions in a different manner than appetitive learning. In active avoidance, DLS lesions selectively impair the early stage of acquisition of an active avoidance task, while DMS lesions selectively impair the late stage of learning (Wendler et al., 2014). Similarly, in fear conditioning, DMS appears to be particularly involved in late acquisition whereas DLS is engaged during both the early and late stages of acquisition (Icenhour et al., 2015).

To explain the differential involvement of DS subregions in aversive learning vs. appetitive learning, we propose the following hypotheses: (1) the DLS may work together with the amygdala to develop cue-outcome associations. With extensive learning, avoidance behaviors may shift to habitual defensive behaviors, which might be under the control of the DMS. (2) The ability of DMS D2 SPNs to respond and drive innate avoidance in the elevated plus-maze and open field (LeBlanc et al., 2020) suggests that DMS activity is required for innate avoidance 
behavior. DMS D2 SPN activity may also play an important role in conditioned avoidance behavior and, with extensive training, becomes engaged as the animal learns successful avoidance strategies.

These hypotheses remain speculative, and to test them, future studies must compare how selective interference with DMS and DLS pathways influence revaluation, the ability to change the outcome value of a CS in an aversive paradigm (Campese et al., 2019). Since changes in outcome value alter behavior when actions are goal-directed but not habit directed, interference with the DMS and DLS should alter performance if they mediate goal-directed action, and not habit action. Overall, the role of specific inputs to these DS regions and the differences between direct and indirect SPN outputs from each are poorly understood in aversive learning. The adaption of chemogenetic and optogenetic techniques that selectively target or report from specific cell populations in DS subregions

\section{REFERENCES}

Almada, R. C., Genewsky, A. J., Heinz, D. E., Kaplick, P. M., Coimbra, N. C., and Wotjak, C. T. (2018). Stimulation of the nigrotectal pathway at the level of the superior colliculus reduces threat recognition and causes a shift from avoidance to approach behavior. Front. Neural Circuits 12:36. doi: 10.3389/fncir.2018. 00036

Arbuthnott, G. W., Ingham, C. A., and Wickens, J. R. (2000). Dopamine and synaptic plasticity in the neostriatum. J. Anat. 196, 587-596. doi: 10.1046/j. 1469-7580.2000.19640587.x

Baeuchl, C., Meyer, P., Hoppstädter, M., Diener, C., and Flor, H. (2015). Contextual fear conditioning in humans using feature-identical contexts. Neurobiol. Learn. Mem. 121, 1-11. doi: 10.1016/j.nlm.2015.03.001

Beckstead, R. M., Domesick, V. B., and Nauta, W. J. H. (1979). Efferent connections of the substantia nigra and ventral tegmental area in the rat. Brain Res. 175, 191-217. doi: 10.1016/0006-8993(79)91001-1

Bello-Medina, P. C., Flores, G., Quirarte, G. L., McGaugh, J. L., and Prado Alcalá, R. A. (2016). Mushroom spine dynamics in medium spiny neurons of dorsal striatum associated with memory of moderate and intense training. Proc. Natl. Acad. Sci. U S A 113, E6516-E6525. doi: 10.1073/pnas.1613680113

Bertran-Gonzalez, J., Hervé, D., Girault, J. A., and Valjent, E. (2010). What is the degree of segregation between striatonigral and striatopallidal projections. Front. Neuroanat. 4:136. doi: 10.3389/fnana.2010.00136

Borsook, D., Upadhyay, J., Chudler, E. H., and Becerra, L. (2010). A key role of the basal ganglia in pain and analgesia-insights gained through human functional imaging. Mol. Pain 6:27. doi: 10.1186/1744-8069-6-27

Buhidma, Y., Rukavina, K., Chaudhuri, K. R., and Duty, S. (2020). Potential of animal models for advancing the understanding and treatment of pain in Parkinson's disease. NPJ Park. Dis. 6:1. doi: 10.1038/s41531-019-0104-6

Cain, C. K., and LeDoux, J. E. (2007). Escape from fear: a detailed behavioral analysis of two atypical responses reinforced by CS termination. J. Exp. Psychol. Anim. Behav. Process 33, 451-463. doi: 10.1037/0097-7403.33.4.451

Campese, V. D., Kim, I. T., Hou, M., Gupta, S., Draus, C., Kurpas, B., et al. (2019). Chemogenetic inhibition reveals that processing relative but not absolute threat requires basal amygdala. J. Neurosci. 39, 8510-8516. doi: 10.1523/JNEUROSCI. 2530-18.2019

Castellan-Baldan, L., da Costa Kawasaki, M., Ribeiro, S. J., Calvo, F., Corrêa, V. M. A., and Coimbra, N. C. (2006). Topographic and functional neuroanatomical study of GABAergic disinhibitory striatum-nigral inputs and inhibitory nigrocollicular pathways: neural hodology recruiting the substantia nigra, pars reticulata, for the modulation of the neural activity in the inferior colliculus involved with panic-like emotions. J. Chem. Neuroanat. 32, 1-27. doi: 10.1016/j.jchemneu.2006.05.002

Cefaliello, C., Penna, E., Barbato, C., Di Ruberto, G., Mollica, M. P., Trinchese, G., et al. (2020). Deregulated local protein synthesis in the brain synaptosomes during the different phases of fear conditioning and avoidance behaviors will aid in understanding the DS contribution to aversive learning.

\section{AUTHOR CONTRIBUTIONS}

MCM and DS designed the outline of the article. ATS, PL, MCM, and DS wrote the manuscript. ATS and PL prepared the figures. All authors contributed to the article and approved the submitted version.

\section{FUNDING}

The research related to this field in DS's laboratory was supported by NIH R01 DA07418, R01 MH108186, R01 MH122470, and the JPB Foundation.

of a mouse model for Alzheimer's disease. Mol. Neurobiol. 57, 1529-1541. doi: 10.1007/s12035-019-01835-y

Chavez, M. E., Salado-Castillo, R., Sanchez-Alavez, M., Quirarte, G. L., and PradoAlcala, R. A. (1995). Post-training injection of GABAergic antagonists into the striatum produces retrograde amnesia. Neurobiol. Learn. Mem. 63, 296-300. doi: 10.1006/nlme.1995.1035

Chudler, E. H., and Dong, W. K. (1995). The role of the basal ganglia in nociception and pain. Pain 60, 3-38. doi: 10.1016/0304-3959(94)00172-B

Conzales, C., and Chesselet, M. F. (1990). Amygdalonigral pathway: an anterograde study in the rat with Phaseolus vulgaris leucoagglutinin (PHA-L). J. Comp. Neurol. 297, 182-200. doi: 10.1002/cne.902970203.

DeLong, M. R. (1990). Primate models of movement disorders of basal ganglia origin. Trends Neurosci. 13, 281-285. doi: 10.1016/0166-2236(90)90110-v

Ferreira, T. L., Moreira, K. M., Ikeda, D. C., Bueno, O. F. A., and Oliveira, M. G. M. (2003). Effects of dorsal striatum lesions in tone fear conditioning and contextual fear conditioning. Brain Res. 987, 17-24. doi: 10.1016/s00068993(03)03217-7

Ferreira, T. L., Shammah-Lagnado, S. J., Bueno, O. F. A., Moreira, K. M. Fornari, R. V., and Oliveira, M. G. M. (2008). The indirect amygdaladorsal striatum pathway mediates conditioned freezing: insights on emotional memory networks. Neuroscience 153, 84-94. doi: 10.1016/j.neuroscience.2008. 02.013

Fishbain, D. A., Pulikal, A., Lewis, J. E., and Gao, J. (2017). Chronic pain types differ in their reported prevalence of post-traumatic stress disorder (PTSD) and there is consistent evidence that chronic pain is associated with PTSD: an evidence-based structured systematic review. Pain Med. 18, 711-735. doi: $10.1093 / \mathrm{pm} / \mathrm{pnw} 065$

Fisher, R. S., Levine, M. S., Sibley, D. R., and Ariano, M. A. (1994). D2 dopamine receptor protein location: Golgi impregnation-gold toned and ultrastructural analysis of the rat neostriatum. J. Neurosci. Res. 38, 551-564. doi: 10.1002/jnr. 490380508

Gramsch, C., Kattoor, J., Icenhour, A., Forsting, M., Schedlowski, M., Gizewski, E. R., et al. (2014). Learning pain-related fear: Neural mechanisms mediating rapid differential conditioning, extinction and reinstatement processes in human visceral pain. Neurobiol. Learn. Mem. 116, 36-45. doi: 10.1016/j.nlm.2014.08.003

Green, R. H., Beatty, W. W., and And Schwartzbaum, J. S. (1967). Comparative effects of septo-hippocampal and caudate lesions on avoidance behavior in rats. J. Comp. Physiol. Psychol. 64, 444-452. doi: 10.1037/h0025195

Haber, S. N. (2003). The primate basal ganglia: parallel and integrative networks. J. Chem. Neuroanat. 26, 317-330. doi: 10.1016/j.jchemneu.2003. 10.003

Hasler, G., Fromm, S., Alvarez, R. P., Luckenbaugh, D. A., Drevets, W. C., and Grillon, C. (2007). Cerebral blood flow in immediate and sustained anxiety. J. Neurosci. 27, 6313-6319. doi: 10.1523/JNEUROSCI.5369-06.2007 
Hedreen, J. C., and Delong, M. R. (1991). Organization of striatopallidal, striatonigral, and nigrostriatal projections in the macaque. J. Comp. Neurol. 304, 569-595. doi: 10.1002/cne.903040406

Holschneider, D. P., Yang, J., Sadler, T. R., Nguyen, P. T., Givrad, T. K., and Maarek, J. M. I. (2006). Mapping cerebral blood flow changes during auditory-cued conditioned fear in the nontethered, nonrestrained rat. NeuroImage 29, 1344-1358. doi: 10.1016/j.neuroimage.2005.08.038

Hormigo, S., Vega-Flores, G., and Castro-Alamancos, M. A. (2016). Basal ganglia output controls active avoidance behavior. J. Neurosci. 36, 10274-10284. doi: 10.1523/JNEUROSCI.1842-16.2016

Hunnicutt, B. J., Jongbloets, B. C., Birdsong, W. T., Gertz, K. J., Zhong, H., and Mao, T. (2016). A comprehensive excitatory input map of the striatum reveals novel functional organization. eLife 5:e19103. doi: 10.7554/eLife.19103

Icenhour, A., Kattoor, J., Benson, S., Boekstegers, A., Schlamann, M., Merz, C. J., et al (2015). Neural circuitry underlying effects of context on human pain-related fear extinction in a renewal paradigm. Hum. Brain Mapp. 36, 3179-3193. doi: 10.1002/hbm.22837

Icenhour, A., Labrenz, F., Ritter, C., Theysohn, N., Forsting, M., Bingel, U., et al (2017). Learning by experience-visceral pain-related neural and behavioral responses in a classical conditioning paradigm. Neurogastroenterol. Motil. 29, 1-11. doi: $10.1111 / \mathrm{nmo} .13026$

Ipser, J. C., Singh, L., and Stein, D. J. (2013). Meta-analysis of functional brain imaging in specific phobia. Psychiatry Clin. Neurosci. 67, 311-322. doi: $10.1111 /$ pcn. 12055

Kattoor, J., Gizewski, E. R., Kotsis, V., Benson, S., Gramsch, C., Theysohn, N., et al (2013). Fear conditioning in an abdominal pain model: neural responses during associative learning and extinction in healthy subjects. PLoS One 8:e51149. doi: 10.1371/journal.pone.0051149

Kirkby, R. J., and Kimble, D. P. (1968). Avoidance and escape behavior following striatal lesions in the rat. Exp. Neurol. 20, 215-227. doi: 10.1016/00144886(68)90095-2

Kirkby, R. J., and Polgar, S. (1974). Active avoidance in the laboratory rat following lesions of the dorsal or ventral caudate nucleus. Physiol. Psychol. 2, 301-306. doi: 10.3758/bf03333025

Koolhaas, J. M., Korte, S. M., De Boer, S. F., Van Der Vegt, B. J., Van Reenen, C. G., Hopster, H., et al (1999). Coping styles in animals: current status in behavior and stress-physiology. Neurosci. Biobehav. Rev. 23, 925-935. doi: 10.1016/s0149-7634(99)00026-3

Koyama, T., Kato, K., and Mikami, A. (2000). During pain-avoidance neurons activated in the macaque anterior cingulate and caudate. Neurosci. Lett. 283, 17-20. doi: 10.1016/s0304-3940(00)00894-6

Krabbe, S., Gründemann, J., and Lüthi, A. (2018). Amygdala inhibitory circuits regulate associative fear conditioning. Biol. Psychiatry 83, 800-809. doi: 10.1016/j.biopsych.2017.10.006

Krout, K. E., Loewy, A. D., Max Westby, G. W., and Redgrave, P. (2001). Superior colliculus projections to midline and intralaminar thalamic nuclei of the rat. J. Comp. Neurol. 431, 198-216. doi: 10.1002/10969861(20010305)431:2<198::aid-cne1065>3.0.co;2-8

LeBlanc, K. H., London, T. D., Szczot, I., Bocarsly, M. E., Friend, D. M., Nguyen, K. P., et al (2020). Striatopallidal neurons control avoidance behavior in exploratory tasks. Mol. Psychiatry 25, 491-505. doi: 10.1038/s41380-0180051-3

LeDoux, J. E. (2000). Emotion circuits in the brain. Annu. Rev. Neurosci. 23, 155-184. doi: 10.1146/annurev.neuro.23.1.155

LeDoux, J. E., Iwata, J., Cicchetti, P., and Reis, D. J. (1988). Different projections of the central amygdaloid nucleus mediate autonomic and behavioral correlates of conditioned fear. J. Neurosci. 8, 2517-2529. doi: 10.1523/JNEUROSCI.0807-02517.1988

LeDoux, J. E., Moscarello, J., Sears, R., and Campese, V. (2017). The birth, death and resurrection of avoidance: a reconceptualization of a troubled paradigm. Mol. Psychiatry 22, 24-36. doi: 10.1038/mp. 2016.166

LeDoux, J. E., Ruggiero, D. A., and Reis, D. J. (1985a). Projections to the subcortical forebrain from anatomically defined regions of the medial geniculate body in the rat. J. Comp. Neurol. 242, 182-213. doi: 10.1002/cne.902420204

LeDoux, J. E., Sakaguchi, A., Iwata, J., and Reis, D. J. (1985b). Auditory emotional memories: establishment by projections from the medial geniculate nucleus to the posterior neostriatum and/or dorsal amygdala. Ann. N. Y. Acad. Sci. 444, 463-464. doi: 10.1111/j.1749-6632.1985.tb37611.x

LeDoux, J. E., Sakaguchi, A., and Reis, D. J. (1984). Subcortical efferent projections of the medial geniculate nucleus mediate emotional responses conditioned to acoustic stimuli. J. Neurosci. 4, 683-698. doi: 10.1523/JNEUROSCI.04-0300683.1984

LeDoux, J. E., Sakaguchi, A., Iwata, J., and Reis, D. J. (1986). Interruption of projections from the medial geniculate body to an archi-neostriatal field disrupts the classical conditioning of emotional responses to acoustic stimuli. Neuroscience 17, 615-627. doi: 10.1016/0306-4522(86) 90034-5

Lenz, J. D., and Lobo, M. K. (2013). Optogenetic insights into striatal function and behavior. Behav. Brain Res. 255, 44-54. doi: 10.1016/j.bbr.2013.04.018

Li, P., Li, Y. H., and Han, T. Z. (2009). NR2A-containing NMDA receptors are required for LTP induction in rat dorsolateral striatum in vitro. Brain Res. 1274, 40-46. doi: 10.1016/j.brainres.2009.04.016

Linke, R. (1999). Differential projection patterns of superior and inferior collicular neurons onto posterior paralaminar nuclei of the thalamus surrounding the medial geniculate body in the rat. Eur. J. Neurosci. 11, 187-203. doi: 10.1046/j.1460-9568.1999. 00422.x

MacKay-Lyons, M. (2002). Central pattern generation of locomotion: a review of the evidence. Phys. Ther. 82, 69-83. doi: 10.1093/ptj/82.1.69

Maren, S. (2001). Neurobiology of Pavlovian fear conditioning. Annu. Rev. Neurosci. 24, 897-931. doi: 10.1146/annurev.neuro.24.1.897

Moscarello, J. M., and LeDoux, J. E. (2013). Active avoidance learning requires prefrontal suppression of amygdala-mediated defensive reactions. J. Neurosci. 33, 3815-3823. doi: 10.1523/JNEUROSCI.2596-12.2013

Nader, K., Majidishad, P., Amorapanth, P., and LeDoux, J. E. (2001). Damage to the lateral and central, but not other, amygdaloid nuclei prevents the acquisition of auditory fear conditioning. Learn. Mem. 8, 156-163. doi: $10.1101 / \mathrm{lm} .38101$

Packard, M. G., and Knowlton, B. J. (2002). Learning and memory functions of the basal ganglia. Annu. Rev. Neurosci. 25, 563-593. doi: 10.1146/annurev.neuro. 25.112701.142937

Phelps, E. A., and LeDoux, J. E. (2005). Contributions of the amygdala to emotion processing: from animal models to human behavior. Neuron 48, 175-187. doi: 10.1016/j.neuron.2005.09.025

Ponvert, N. D., and Jaramillo, S. (2019). Thalamostriatal and corticostriatal pathways convey complementary information about sound features. J. Neurosci. 39, 271-280. doi: 10.1523/JNEUROSCI.1188-18.2018

Powell, D. A., Mankowski, D., and Buchanan, S. (1978). Concomitant heart rate and corneoretinal potential conditioning in the rabbit (Oryctolagus Cuniculus): effects of caudate lesions. Physiol. Behav. 20, 143-150. doi: 10.1016/00319384(78)90066-5

Prado-Alcalá, R. A., Grinberg, Z. J., Arditti, Z. L., García, M. M., Prieto, H. G., and Brust-Carmona, H. (1975). Learning deficits produced by chronic and reversible lesions of the corpus striatum in rats. Physiol. Behav. 15, 283-287. doi: 10.1016/0031-9384(75)90095-5

Prado-Alcalá, R. A., Medina, A. C., Bello-Medina, P. C., and Quirarte, G. L. (2017). Inhibition of transcription and translation in the striatum after memory reactivation: lack of evidence of reconsolidation. Neurobiol. Learn. Mem. 142, 21-29. doi: 10.1016/j.nlm.2016.12.018

Sah, P., Sun, Y., and Gooch, H. (2020). Fear conditioning and the basolateral amygdala. F1000Research 9:53. doi: 10.12688/f1000research.21201.1

Sanders, M. J., Wiltgen, B. J., and Fanselow, M. S. (2003). The place of the hippocampus in fear conditioning. Eur. J. Pharmacol. 463, 217-223. doi: 10.1016/s0014-2999(03)01283-4

Schenberg, E. E., Ferreira, T. L., Figueredo, L. Z. P., Hipólide, D. C., Nobrega, J. N., and Oliveira, M. G. M. (2006). Fear conditioning performance and NMDA receptor subtypes: NR2A differential expression in the striatum. Brain Res. Bull. 69, 440-446. doi: 10.1016/j.brainresbull.2006.02.010

Sripada, R. K., Garfinkel, S. N., and Liberzon, I. (2013). Avoidant symptoms in PTSD predict fear circuit activation during multimodal fear extinction. Front. Hum. Neurosci. 7:672. doi: 10.3389/fnhum.2013.00672

Tovote, P., Fadok, J. P., and Lüthi, A. (2015). Neuronal circuits for fear and anxiety. Nat. Rev. Neurosci. 16, 317-331. doi: 10.1038/nrn3945 
Vetere, G., Kenney, J. W., Tran, L. M., Xia, F., Steadman, P. E., Parkinson, J., et al (2017). Chemogenetic interrogation of a brain-wide fear memory network in mice. Neuron 94, 363-374. doi: 10.1016/j.neuron.2017.03.037

Viaud, M. D., and White, N. M. (1989). Dissociation of visual and olfactory conditioning in the neostriatum of rats. Behav. Brain Res. 32, 31-42. doi: 10.1016/s0166-4328(89)80069-5

Wendler, E., Gaspar, J. C. C., Ferreira, T. L., Barbiero, J. K., Andreatini, R., Vital, M. A. B. F., et al (2014). The roles of the nucleus accumbens core, dorsomedial striatum and dorsolateral striatum in learning: performance and extinction of Pavlovian fear-conditioned responses and instrumental avoidance responses. Neurobiol. Learn. Mem. 109, 27-36. doi: 10.1016/j.nlm.2013.11. 009

Wheeler, A. L., Teixeira, C. M., Wang, A. H., Xiong, X., Kovacevic, N., Lerch, J. P., et al (2013). Identification of a functional connectome for long-term fear memory in mice. PLoS Comput. Biol. 9:e1002853. doi: 10.1371/journal.pcbi. 1002853

White, N. M., and Salinas, J. A. (2003). Mnemonic functions of dorsal striatum and hippocampus in aversive conditioning. Behav. Brain Res. 142, 99-107. doi: 10.1016/s0166-4328(02)00402-3

White, N. M., and Viaud, M. (1991). Localized intracaudate dopamine D2 receptor activation during the post-training period improves memory for visual or olfactory conditioned emotional responses in rats. Behav. Neural Biol. 55, 255-269. doi: 10.1016/0163-1047(91)90609-t

Wood, S. K., and Bhatnagar, S. (2015). Resilience to the effects of social stress: evidence from clinical and preclinical studies on the role of coping strategies. Neurobiol. Stress 1, 164-173. doi: 10.1016/j.ynstr.2014.11.002

Yin, H. H., Knowlton, B. J., and Balleine, B. W. (2006). Inactivation of dorsolateral striatum enhances sensitivity to changes in the action-outcome contingency in instrumental conditioning. Behav. Brain Res. 166, 189-196. doi: 10.1016/j.bbr. 2005.07.012

Conflict of Interest: The authors declare that the research was conducted in the absence of any commercial or financial relationships that could be construed as a potential conflict of interest.

Copyright (C) 2021 Stanley, Lippiello, Sulzer and Miniaci. This is an open-access article distributed under the terms of the Creative Commons Attribution License (CC BY). The use, distribution or reproduction in other forums is permitted, provided the original author(s) and the copyright owner(s) are credited and that the original publication in this journal is cited, in accordance with accepted academic practice. No use, distribution or reproduction is permitted which does not comply with these terms. 